\title{
Модуляция заряда германиевых МДП-структур с фторсодержащими диэлектриками
}

\author{
(C) М.Б. Шалимова
}

Самарский национально-исследовательский университет, 443011 Самара, Россия

E-mail: shamb@ssau.ru

(Получена 28 сентября 2017 г. Принята к печати 18 октября 2017 г.)

В МДП-структурах на основе германия $n$-типа формировался диэлектрический слой из $\mathrm{ErF}_{3}, \mathrm{YF}_{3}$, $\mathrm{NdF}_{3}$ и $\mathrm{TmF}_{3}$. Показано, что отрицательный эффективный заряд в данных германиевых МДП-структурах не наблюдается, а деградация электрических характеристик и рост плотности поверхностных состояний приводят к увеличению положительного заряда. Положительный заряд, создаваемый на границе раздела германий-фторид редкоземельного элемента, может компенсировать отрицательный заряд оборванных связей на поверхности германия, что потенциально перспективно для модуляции величины и знака заряда в МДП-устройствах на германии.

DOI: 10.21883/FTP.2018.08.46223.8738

\section{1. Введение}

Поиск новых материалов для полевых транзисторов металл-диэлектрик-полупроводник (МДП) вызван тем, что миниатюризация устройств накладывает жесткие ограничения на размер элемента, поэтому удовлетворительная производительность устройства не может быть больше гарантирована основанной на кремнии технологией. Деградация подвижности носителей заряда, вызванная уменьшением длины затвора, может быть нивелирована использованием преимущества более высокой скорости носителей заряда в канале полевого транзистора. Одной из основных причин повышенного научного интереса к германию является в 4 раза большая дырочная и в 2 раза большая электронная подвижность носителей в объемном германии по сравнению с подложками $\mathrm{Si}$. В этой связи актуальной проблемой является интеграция $\mathrm{Ge}$ в качестве альтернативного канального материала в технологию изготовления высокоскоростных МДП-устройств и сопутствующего затворного диэлектрика с высокой диэлектрической проницаемостью (high- $k$ ).

Пассивация германиевых поверхностей с использованием high- $k$ диэлектриков проводится для улучшения электрических свойств МДП-устройств. Разнообразные способы пассивации поверхности германия представлены в литературе: метод молекулярно-лучевого осаждения пленок $\mathrm{Gd}_{2} \mathrm{O}_{3}$ на подложках $\mathrm{Ge}$ c последующим послойным атомным осаждением $\mathrm{HfO}_{2}$ [1]; атомное послойное осаждение high- $k$ диэлектриков $\mathrm{La}_{2} \mathrm{O}_{3} / \mathrm{ZrO}_{2}$ [2]; электроннолучевое испарение high- $k$ диэлектриков $\mathrm{Y}_{2} \mathrm{O}_{3}, \mathrm{HfO}_{2}$ и $\mathrm{Ga}_{2} \mathrm{O}_{3}\left(\mathrm{Gd}_{2} \mathrm{O}_{3}\right)$ в сверхвысоком вакууме на подложки $\mathrm{Ge}$ [3]. МДП конденсаторы на основе германия c high- $k$ многослойной затворной структурой $\mathrm{GeO}_{2} / \mathrm{Al}_{2} \mathrm{O}_{3} / \mathrm{HfO}_{2}$ представляли хорошие электрические характеристики $[4,5]$. Изготавливались $\mathrm{Ge}$ МДП конденсаторы с диэлектриком HfTiON, где для пассивации поверхности фтор внедрялся перед осаждением в процессе предварительной обработки поверхно- сти $\mathrm{Ge}$, или в процессе отжига после осаждения, в обоих случаях получено улучшение электрических свойств [6].

В процессе работы германиевых МДП-устройств может происходить деградация их электрофизических параметров, что уменьшает первоначальную эффективность пассивации. Наблюдения показывают, что структура диэлектрика может быть модифицирована путем приложенного электрического „стресса“ (электрической перегрузки), что указывает на наличие процессов трансформации дефектов в затворном диэлектрике и на границе раздела диэлектрик-германий. Изменение концентрации электрически активных дефектов, способных захватывать и высвобождать заряд, приводит к нежелательному дрейфу параметров МДП-устройств и уменьшению их срока службы.

Поиск путей стабилизации зарядового состояния МДП-устройств на основе германия является актуальной задачей, требующей как технологического, так и научного решения, поскольку в настоящее время не ясна природа дефектов в затворном диэлектрике и на границе раздела диэлектрик-германий. В связи с этим в данной работе был сформирован диэлектрический слой из фторидов редкоземельных элементов (РЗЭ) на поверхности германия с целью изучения изменения свойств данных МДП-структур в процессе эксплуатации.

\section{2. Экспериментальные образцы}

В данной работе в качестве подложек использовались монокристаллические пластины германия $n$-типа ориентации (111) с удельным сопротивлением 2 Ом · см. Тонкие пленки фторидов редкоземельных элементов получались методом термического распыления в вакууме порошкообразных $\mathrm{ErF}_{3}, \mathrm{YF}_{3}, \mathrm{NdF}_{3}$ и $\mathrm{TmF}_{3}$ на подогретые до $\sim 300^{\circ} \mathrm{C}$ подложки. Толщина слоя $(d)$ фторида РЗЭ, наносимого на германиевую подложку, варьировалась от 0.1 до 0.4 мкм. Оптимальными для изучения процессов деградации в МДП-структуре методом электроформовки являются толщины $0.25-0.40$ мкм. 
Впоследствии на фторид РЗЭ наносились алюминиевые контакты диаметром 0.5-0.7 мм.

Полученные пленочные фториды РЗЭ обладали достаточно большим значением диэлектрической проницаемости $k=14,13,12$ и 7 для пленок $\mathrm{ErF}_{3}, \mathrm{YF}_{3}, \mathrm{NdF}_{3}$ и $\mathrm{TmF}_{3}$ соответственно.

Исследование с помощью атомно-силового микроскопа поверхностной морфологии пленок фторидов РЗЭ показало, что во всех случаях пленка представляет собой совокупность отдельных кристаллов размером $\sim 60-120$ нм, ориентированных в преимущественном направлении.

\section{3. Исследование электрофизических Свойств}

Для изучения условий возникновения дефектов на границе раздела диэлектрик-германий проводился комплексный мониторинг изменения свойств германиевых МДП-структур с данными диэлектриками в условиях повышенных электрических полей. Для этого исследовалась деградация электрофизических свойств германиевых МДП-структур с пленками $\mathrm{ErF}_{3}, \mathrm{YF}_{3}, \mathrm{NdF}_{3}$ и $\mathrm{TmF}_{3}$ при электрической перегрузке (электроформовке), в процессе которой на структуры действовали электрические поля порядка $10^{8} \mathrm{~B} / \mathrm{M}$.

Процесс электроформовки состоит в том, что под действием электрического поля в диэлектрике создается канал проводимости. Однако это состояние не является необратимым, и при обратной полярности прикладываемого напряжения имелась возможность переключения исследуемой структуры обратно в диэлектрическое состояние. При этом была использована возможность многократного переключения структур из диэлектрического состояния в проводящее и обратно, так как данные МДП-структуры с пленками фторидов РЗЭ обладают свойством бистабильного переключения [7]. После каждого цикла электроформовки структура возвращается в диэлектрическое состояние с новой плотностью поверхностных состояний и с новым значением эффективного заряда. Таким образом, получается более широкий ряд экспериментальных данных.

Электрофизические характеристики исследуемых структур измерялись на частоте 1 МГц при комнатной температуре. На рис. 1 показаны $C-V$-характеристики структуры $\mathrm{Al}-\mathrm{TmF}_{3}-n-\mathrm{Ge}$ в исходном состоянии $(0)$ и после нескольких циклов электроформовки.

После нескольких циклов электроформовки вольтфарадные характеристики более растянуты по оси напряжений, что указывает на рост плотности поверхностных состояний. Кроме того, при отрицательных напряжениях появляется „ступенька“, что указывает на более медленное изменение емкости из-за перезарядки поверхностных состояний. В данном случае с ростом числа циклов электроформовки $C-V$-кривые смещаются влево, что указывает на возрастание положительного заряда и изменение напряжения плоских зон.

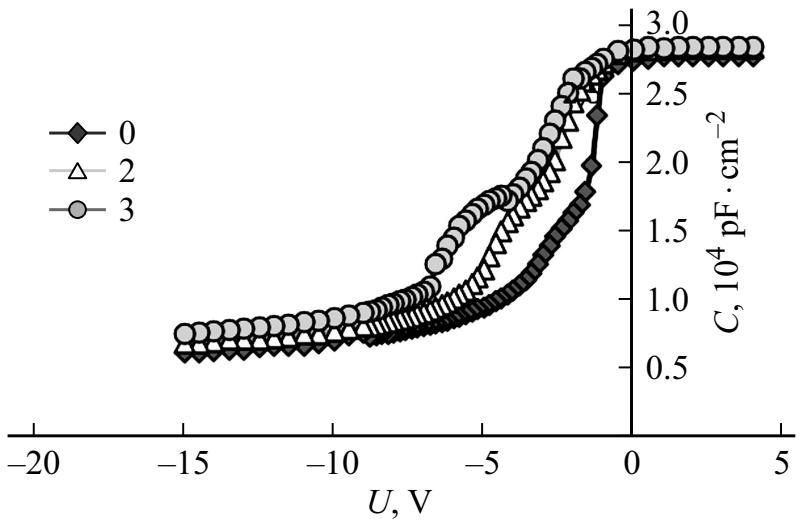

Рис. 1. Вольт-фарадные характеристики структуры $\mathrm{Al}-\mathrm{TmF}_{3}-n-\mathrm{Ge}$ в исходном состоянии и после нескольких циклов электроформовки (число циклов указано на рисунке).

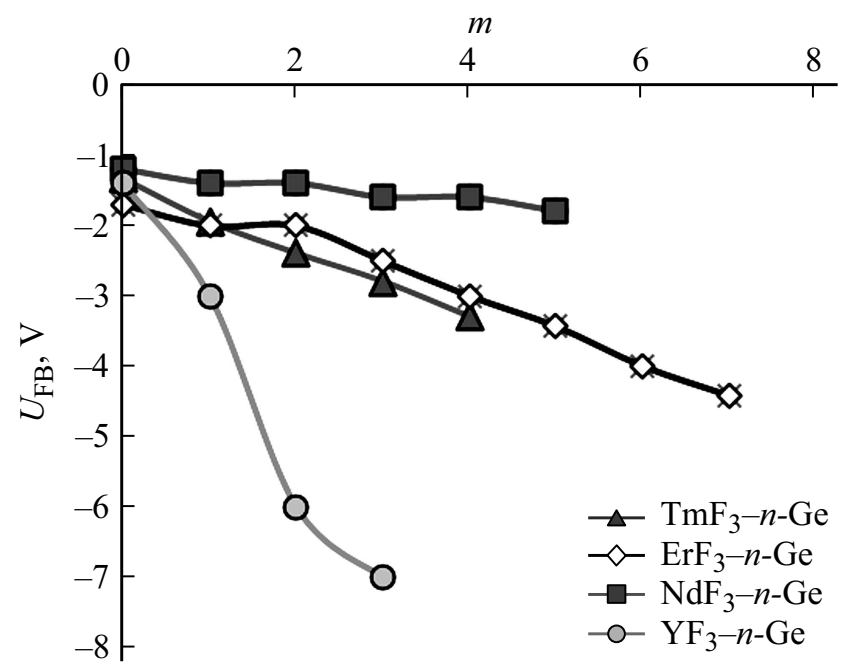

Рис. 2. Изменение напряжения плоских зон при множественных циклах электроформовки для МДП-структур с фторидами P3Э на основе $n$-Ge.

Напряжение плоских зон $U_{\mathrm{FB}}$ - это напряжение, при котором поверхностный потенциал принимает нулевое значение.

$$
U_{\mathrm{FB}}=\Phi_{m s}-\frac{Q_{f}}{C_{D}},
$$

где $\Phi_{m s}-$ разность работ выхода металл-полупроводник, $C_{D}-$ емкость фторида, $Q_{f}-$ эффективный заряд. Под эффективным зарядом здесь понимается суммарная плотность заряда ловушек на границе раздела фторид-германий $Q_{\text {it }}$ и в объеме фторида $Q_{f}^{*}$ :

$$
Q_{f}=Q_{f}^{*}+Q_{\mathrm{it}} .
$$

На рис. 2 показан пример изменения напряжения плоских зон при множественных циклах электроформовки для МДП-структур с фторидами РЗЭ на основе $n$-Ge, где по горизонтальной оси откладывается номер цикла электроформовки. На представленных зависимостях наблюдается сдвиг напряжения плоских зон в область 


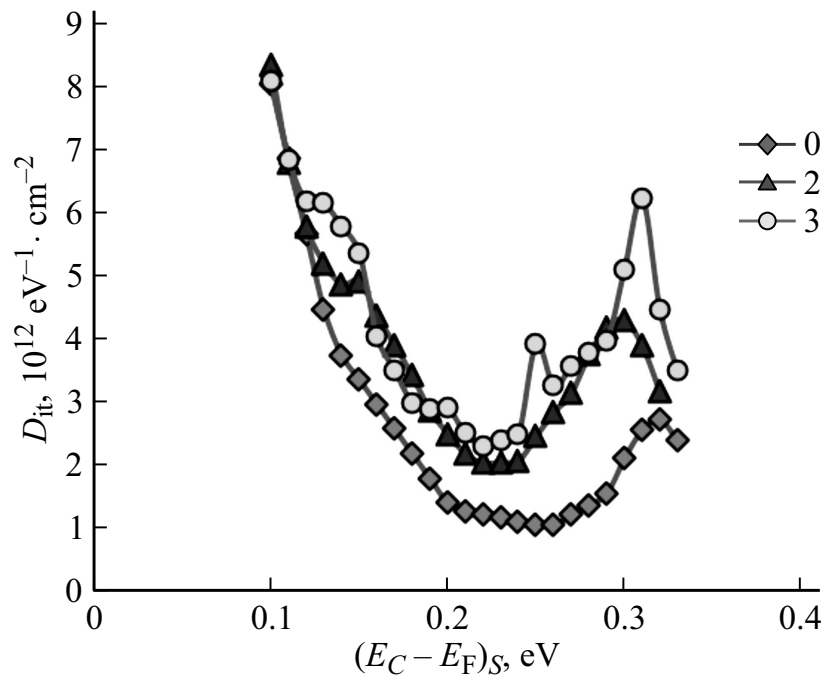

Рис. 3. Распределение плотности поверхностных состояний по энергии в запрещенной зоне германия при нескольких циклах электроформовки для структуры $\mathrm{Al}-\mathrm{TmF}_{3}-n-\mathrm{Ge}$, номер цикла указан.

отрицательных значений при росте числа циклов, что указывает на рост положительного заряда.

Распределение энергетической плотности поверхностных состояний $D_{\text {it }}$ в запрещенной зоне германия рассчитывалось из зависимости поверхностного потенциала от напряжения $U$ по формуле

$$
D_{\mathrm{it}}=\frac{C_{D}}{e^{2}}\left(\frac{d U}{d \varphi_{S}}-1\right)+\frac{C_{s}}{e^{2}},
$$

где $C_{s}$ - емкость области пространственного заряда, $\varphi_{S}$ - величина поверхностного потенциала, $e-$ элементарный заряд. На рис. 3 представлены данные расчета по формуле (3) для структуры $\mathrm{Al}-\mathrm{TmF}_{3}-n-\mathrm{Ge}$, где $E_{C}$ и $E_{\mathrm{F}}$ - энергия дна зоны проводимости германия и энергия уровня Ферми соответственно. Данный метод дает $U$-образный вид энергетического распределения $D_{\text {it }}$ в запрещенной зоне германия для исследованных структур. В качестве значения $D_{\text {it }}$ в настоящей работе принималось значение в минимуме распределения.

Зависимость эффективного заряда $Q_{f}$ от плотности поверхностных состояний $D_{\text {it }}$ при множественных циклах электроформовки показана на рис. 4 для германиевых МДП-структур с пленками $\mathrm{NdF}_{3}$ и $\mathrm{YF}_{3}$. Аналогичные зависимости для структур $\mathrm{Al}-\mathrm{ErF}_{3}-n-\mathrm{Ge}$ и $\mathrm{Al}-\mathrm{TmF}_{3}-n-\mathrm{Ge}$ показаны на рис. 5. Во всех случаях наблюдается тенденция роста положительного заряда при росте плотности поверхностных состояний для структур на основе германия $n$-типа.

Исследования показывают, что отрицательный эффективный заряд в данных германиевых МДП-структурах не наблюдается, а деградация электрических характеристик и рост плотности поверхностных состояний приводят к увеличению положительного заряда. Это отличается от случая оксидов РЗЭ на кремнии $n$-типа, когда эффективный заряд может быть как положительным, так и отрицательным [8].

Ранее было показано с помощью теоретических расчетов из первых принципов $[9,10]$, что оборванные связи германия на его поверхности выстраивают отрицательный заряд независимо от положения уровня Ферми в запрещенной зоне.

В работе [4] на подложках германия были изготовлены конденсаторы металл-оксид-полупроводник (МОП) с многослойной структурой high- $k$ затвора $\mathrm{GeO}_{2} / \mathrm{Al}_{2} \mathrm{O}_{3}$ и $\mathrm{GeO}_{2} / \mathrm{HfO}_{2}$. Для проверки термической стабильности слоев high- $k$ затвора использовался отжиг после металлизации, т.е. обработка в окружающей среде $\mathrm{N}_{2}$ или $\mathrm{N}_{2} / \mathrm{H}_{2}$ в течение 30 мин при температуре от 300 до $450^{\circ} \mathrm{C}$. После обработки напряжение плоских зон сдвигалось к положительным значениям, что указывало на рост отрицательного заряда. Сдвиг $C-V$-характеристик в положительную область напряжений увеличивался с

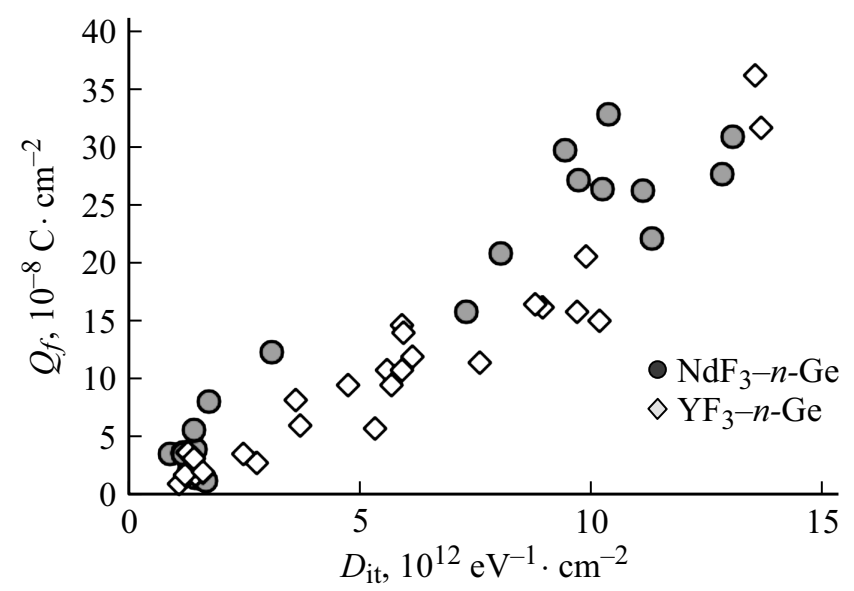

Рис. 4. Изменение эффективного заряда в зависимости от изменения плотности поверхностных состояний для МДП-структур с фторидами неодима и иттрия на основе $n$-Ge.

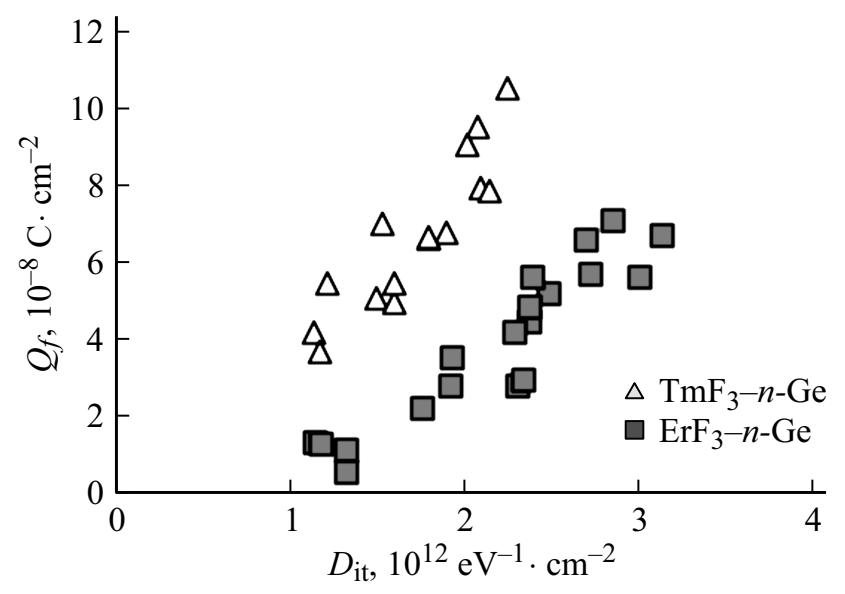

Рис. 5. Изменение эффективного заряда в зависимости от изменения плотности поверхностных состояний для МДП-структур с фторидами тулия и эрбия на основе $n$-Ge. 
ростом температуры обработки от 300 до $450^{\circ} \mathrm{C}$ в окружающей среде $\mathrm{N}_{2}$. Максимальный сдвиг $C-V$-характеристик в положительную область происходил при обработке в среде $\mathrm{N}_{2} / \mathrm{H}_{2}$ при $400^{\circ} \mathrm{C}$.

В работе [3] проводился анализ электрических характеристик конденсаторов c high- $k$ диэлектриками $\mathrm{Y}_{2} \mathrm{O}_{3}$, $\mathrm{HfO}_{2}$ и $\mathrm{Ga}_{2} \mathrm{O}_{3}\left(\mathrm{Gd}_{2} \mathrm{O}_{3}\right)$, которые были непосредственно осаждены на подложку $\mathrm{Ge}$ с помощью электроннолучевого испарения в ультравысоком вакууме. Часть образцов обрабатывалась плазмой $\mathrm{CF}_{4}$ в течение 3 и 5 мин с последующим отжигом при $500^{\circ} \mathrm{C}$ в течение 5 мин в азоте. При этом $U_{\mathrm{FB}}$ сдвигалось к отрицательным значениям по мере увеличения времени обработки. Было замечено, что отрицательный сдвиг $U_{\mathrm{FB}}$ может быть вызван внедрением ионов фтора и небольшим дефицитом кислорода, так как небольшое количество фторида гадолиния образуется вблизи поверхности оксида. Присутствие $\mathrm{F}^{-1}$ в месте расположения $\mathrm{O}^{-2}$ может способствовать наличию положительных зарядов.

Аналогичный эффект возрастания положительного заряда в германиевой МДП-структуре с пленкой HfTiON наблюдался в работе [6] после обработки плазмой $\mathrm{CHF}_{3}$ при $5^{\circ} \mathrm{C}$ в течение 300 с по сравнению с контрольным образцом, необработанным плазмой. Было сделано предположение, что включения фтора могут связываться с оборванными связями Hf, тем самым уменьшая отрицательные заряды.

В настоящее время актуальной задачей для МДПтранзисторов на основе Ge является поиск высококачественных и термодинамически стабильных диэлектриков, которые могут эффективно пассивировать ловушки на границе раздела. Наличие отрицательного заряда на оборванных связях создает серьезную проблему для устройств, работа которых основана на формировании электронного канала, таких как $n$-канальные МДП-транзисторы. Это также приводит к нежелательному рассеиванию носителей в германиевых МДП-устройствах с $p$-каналом и вызывает сдвиг положительного порогового напряжения.

В кремниевых устройствах для пассивации оборванных связей обычно используется гидрирование. Было обнаружено, однако, что водородная пассивация оборванных связей в $\mathrm{Ge}$ является неэффективной, так как электростатическое отталкивание между оборванной связью и отрицательно заряженным интерстициальным Н будет подавлять пассивацию, что объясняет экспериментальные наблюдения, которые не показывают никаких улучшений в производительности германиевого МДП-устройства при гидрогенизации [9].

Настоящие исследования показали, что пассивация поверхности германия диэлектрическими пленками фторидов $\mathrm{ErF}_{3}, \mathrm{YF}_{3}, \mathrm{NdF}_{3}$ и $\mathrm{TmF}_{3}$ приводит к положительному эффективному заряду. При росте числа оборванных связей на поверхности германия в результате электрических перегрузок, повышенных температур одновременно растет и положительный заряд на границе раздела, что может быть использовано для стабилизации зарядового состояния МДП-устройств. Вопрос о причинах положительного заряда в германиевых МДП-структурах с пленками $\mathrm{ErF}_{3}, \mathrm{YF}_{3}, \mathrm{NdF}_{3}$ и $\mathrm{TmF}_{3}$ остается открытым и требует дальнейшего изучения. Положительный заряд, создаваемый на границе раздела германий-фторид Р3Э, может компенсировать отрицательный заряд оборванных связей на поверхности германия, что потенциально перспективно для модуляции величины и знака заряда в МДП-устройствах на германии.

\section{4. Заключение}

Комплексное изучение условий возникновения дефектов, которые могут присутствовать в диэлектрике вблизи границы раздела, или на самой границе раздела германий-диэлектрик, имеет большое значение для поиска и прогнозирования затворных материалов с улучшенными качествами и в конечном счете улучшения производительности устройств. В данной работе в МДП-устройствах на основе германия формировался диэлектрический слой из $\mathrm{ErF}_{3}, \mathrm{YF}_{3}, \mathrm{NdF}_{3}$ и $\mathrm{TmF}_{3}$ с целью стабилизации зарядового состояния устройств. Показано, что отрицательный эффективный заряд в данных германиевых МДП-структурах не наблюдается, а деградация электрических характеристик и рост плотности поверхностных состояний приводят к увеличению положительного заряда. Положительный заряд, создаваемый на границе раздела германий-фторид РЗЭ, может компенсировать отрицательный заряд оборванных связей на поверхности германия, что потенциально перспективно для модуляции величины и знака заряда в МДП-устройствах на германии.

\section{Список литературы}

[1] A. Molle, S. Baldovino, S. Spiga, M. Fanciulli. Thin Solid Films, 518, S96 (2010).

[2] B.C. Henkel, P-E. Hellstroöm, M. Östling, M. Stöger-Pollach, O. Bethge, E. Bertagnolli. Solid-State Electron., 74, 7 (2012).

[3] L.K. Chu, R.L. Chu, T.D. Lin, W.C. Lee, C.A. Lin, M.L. Huang, Y.J. Lee, J. Kwo, M. Hong. Solid-State Electron., 54, 965 (2010).

[4] S.N.A. Murad, P.T. Baine, D.W. McNeill, S.J.N. Mitchell, B.M. Armstrong, M. Modreanu, G. Hughes, R.K. Chellappan. Solid-State Electron., 78, 136 (2012).

[5] S. Mather, N. Sedghi, M. Althobaiti, I.Z. Mitrovic, V. Dhanak, P.R. Chalker, S. Hall. Microelectron. Engin., 109, 126 (2013).

[6] C.X. Li, C.D. Wanga, C.H. Leung, P.T. Lai, J.P. Xu. Microelectron. Engin., 86, 1596 (2009).

[7] M.B. Shalimova, E.N. Khavdey. Chapter in the book Germanium: Properties, Production and Applications (N.Y., NOVA, 2012) p. 187.

[8] М.Б. Шалимова, Н.В. Сачук, ФТП, 49, 1071 (2015).

[9] C.G. Van de Walle, M. Choi, J.R. Weber, J.L. Lyons, A. Janotti. Microelectron. Engin., 109, 211 (2013).

[10] P. Tsipas, A. Dimoulas. Appl. Phys. Lett., 94, 012114 (2009).

Редактор А.Н. Смирнов 


\section{Modulation of the charge of germanium \\ MIS structures with fluorine-containing \\ dielectrics}

M.B. Shalimova

Samara National Research University, 443011 Samara, Russian Federation

Abstract In the MIS-structures based on $n$-type germanium, a dielectric layer of $\mathrm{ErF}_{3}, \mathrm{YF}_{3}, \mathrm{NdF}_{3}$ and $\mathrm{TmF}_{3}$ was formed. It is shown that a negative effective charge in these germanium MIS-structures is not observed, and degradation of electrical characteristics and an increase in the density of surface states lead to an increase in the positive charge. The positive charge created at the germanium-fluoride REE interface can compensate for the negative charge of broken bonds on the germanium surface, which is potentially promising for modulating the magnitude and sign of charge in MIS-devices on germanium. 